\title{
Effects of Bifidobacteriumlongumbv.infantisCCUG 52486 combined with glucooligosaccharideon immune cell populations in healthy young and older subjects receiving an influenza vaccination
}

\author{
A. Przemska, C. Maidens, C. Childs, H. Dong, K. Tuohy, I. Rowland, M. Gosney, S. Todd \\ and P. Yaqoob \\ University of Reading, Department of Food and Nutritional Sciences, Reading RG6 6AP. *Nutrition and Nutrigenomics \\ Group, Research and Innovation Centre-Fondazione Edmund Mach, San Michele all'Adige, Trento, Italy
}

Probiotics have been suggested to modulate immune function in young and older subjects. However, the data is inconsistent, the mechanisms are not well characterised, and the influence of immunological ageing is unclear. The aim of the current study was to investigate the impact of a novel probiotic, with a suitable prebiotic, on the immune response to influenza vaccination in healthy young and older subject.

In a randomised, double-blind, placebo-controlled trial, 54 older subjects $(69 \pm 4.6 \mathrm{y})$ and 58 young subjects (26 $\pm 4.2 \mathrm{y})$ consumed either $5 \times 10^{8} \mathrm{CFU}$ of Bifidobacteriumlongumbv.infantisCCUG 52486 combined with $8 \mathrm{~g}$ of glucooligosaccharide (GIOS), or placebo (9 $\mathrm{g}$ maltodextrin) daily for 8 weeks in total. After 4 weeks, subjects received an influenza vaccine (2010/2011 season). Quantification of T cells, NK cells and B cells was performed by flowcytometryat baseline and 4, 6 and 8 weeks after supplementation. Statistical analysis was performed using SPSS software and the Linear Mixed Model ANOVA.

Young subjects had significantly higher number of B cells $(P<0.001)$, and this was particularly evident following vaccination $($ age*time interaction $P<0.05)$. In contrast, older subjects had significantly more NK cells $(P<0.001)$, but there was no change following vaccination. Young subjects had significantly more $\mathrm{T}$ cells compared to older subjects. After vaccination there was a slight increase in $\mathrm{T}$ cell number in the young cohort, but this did not reach statistical significance. Although total numbers of naïve $\mathrm{T}$ cells were unaffected by ageing, numbers of $\mathrm{CD}^{+}$naïve $\mathrm{T}$ cells in older subjects were significantly lower than those in the young subjects. There was no influence of the pre- and probiotic treatment on numbers of immune cell populations.

Our findings confirman influence of ageing on innate and adaptive immunity, chiefly lower $\mathrm{T}$ cell and B cell numbers and elevated numbers of NK cells in older subjects. Although, there was no effect of treatment on the immune cell numbers, we are currently investigating the effects of the pre- and probiotic on activity of NK cells, B cells and $\mathrm{T}$ cells following vaccination. 